\title{
On Generalized Growth of Analytic Functions Solutions of Linear Homogeneous Partial Differential Equation of Second Order
}

\author{
Devendra Kumar ${ }^{1,2}$ \\ ${ }^{1}$ Department of Mathematics, Faculty of Sciences, Al-Baha University, P.O. Box 1988, Al-Aqiq, Al-Baha 65431, Saudi Arabia \\ ${ }^{2}$ Department of Mathematics, Research and Post Graduate Studies, MMH College, Model Town, Ghaziabad 201 001, India
}

Correspondence should be addressed to Devendra Kumar; d_kumar001@rediffmail.com

Received 20 August 2017; Revised 22 October 2017; Accepted 5 November 2017; Published 15 November 2017

Academic Editor: Der-Chen Chang

Copyright (C) 2017 Devendra Kumar. This is an open access article distributed under the Creative Commons Attribution License, which permits unrestricted use, distribution, and reproduction in any medium, provided the original work is properly cited.

\begin{abstract}
The generalized growth parameters of analytic functions solutions of linear homogeneous partial differential equations of second order have been studied. Moreover, coefficients characterizations of generalized order and generalized type of the solutions represented in convergent series of Laguerre polynomials have been obtained.
\end{abstract}

\section{Introduction}

Hu and Yang $[1,2]$ studied the behavior of meromorphic solutions of the following homogeneous linear partial differential equations of the second order:

$$
\begin{gathered}
t^{2} \frac{\partial^{2} u}{\partial t^{2}}-z^{2} \frac{\partial^{2} u}{\partial z^{2}}+(2 t+2) \frac{\partial u}{\partial t}-2 z \frac{\partial u}{\partial z}=0 \\
t^{2} \frac{\partial^{2} u}{\partial t^{2}}-z^{2} \frac{\partial^{2} u}{\partial z^{2}}+t \frac{\partial u}{\partial t}-z \frac{\partial u}{\partial z}+t^{2} u=0
\end{gathered}
$$

They showed that these solutions are closely related to Bessel functions and Bessel polynomials for $(t, z) \in \mathbb{C}^{2}$. Several authors such as Berenstein and Li [3], Hu and Yang [4], $\mathrm{Hu}$ and $\mathrm{Li}$ [5], $\mathrm{Li}$ [6], and $\mathrm{Li}$ and Saleeby [7] have investigated the global solutions of some first-order partial differential equations. McCoy [8] and Kumar [9] studied the approximation of pseudoanalytic functions on the disk and obtained some coefficient and Bernstein-type growth theorems. Kapoor and Nautiyal [10] and Kumar and Basu [11] characterized the order and type of solutions (not necessarily entire solutions) of certain linear partial differential equations in terms of rates of decay of approximation errors in various norms. These solutions are related to Jacobi polynomials. Wang et al. [12] obtained the growth parameters order and type of entire function solutions of the partial differential equation

$$
t \frac{\partial^{2} u}{\partial t^{2}}+(\delta+1-t) \frac{\partial u}{\partial t}+z \frac{\partial u}{\partial z}=0
$$

for a real $\delta>0$. These solutions are related to Laguerre polynomials. In this paper, our aim is to characterize the generalized growth parameters order and type of solutions of (2) which are analytic on bidisk of finite radius $R$. The generalized growth parameters have been studied by several authors such as Šeremeta [13], Shah [14], Srivastava and Kumar [15], and Vakarchuk and Zhir [16, 17]. Wang et al. [12] proved that the $\operatorname{PDE}(2)$ has an entire solution $u=f(t, z)$ on $\mathbb{C}^{2}$, if and only if $u=f(t, z)$ has a series expansion $f(t, z)=\sum_{n=0}^{\infty} a_{n} L_{n}(\delta, t) z^{n}$. Here

$$
L_{n}(\delta, t)=\sum_{k=0}^{n}\left(\begin{array}{l}
n+\delta \\
n-k
\end{array}\right) \frac{(-t)^{k}}{k !}
$$

are the Laguerre polynomials. Bernstein theorem identifies a real analytic function on the closed unit disk as the restriction of an analytic function defined on an open disk of radius $R>1$ by computing $R$ from the sequence of minimal errors generated from optimal polynomials approximates. 
The disk $D_{R}$ of maximum radius on which analytic function $f(t, z)$ exists is denoted by $f(t, z) \in A\left(D_{R} \times D_{R}\right)$. If $f$ is an entire function, it has no singularities in the finite positive $\mathbb{C}^{2}$ plane and write $f \in A\left(\mathbb{C}^{2}\right)$. Let $\alpha$ and $\beta$ be two positive, strictly increasing, and differentiable functions from $(0, \infty) \rightarrow(0, \infty)$, which satisfies the following conditions for every $\gamma>0$ :

$$
\begin{aligned}
& \lim _{x \rightarrow \infty} \frac{\alpha(\gamma x)}{\alpha(x)}=1, \\
& \lim _{x \rightarrow \infty} \frac{\beta(1+x \omega(x))}{\beta(x)}=1, \\
& \lim _{x \rightarrow \infty} \omega(x)=0, \\
& \lim _{x \rightarrow \infty} \frac{d\left(\beta^{-1}(\gamma \alpha(x))\right)}{d(\log x)} \leq b, \\
& \alpha\left(\frac{x}{\beta^{-1}(\gamma \alpha(x))}\right)=(1+0(x)) \alpha(x), \quad x \rightarrow \infty ;
\end{aligned}
$$

here $d(u)$ denotes the differential of $u$. Now we define the generalized order and generalized type of an entire $f(t, z) \in$ $A\left(\mathbb{C}^{2}\right)$ by

$$
\begin{aligned}
& \rho(\alpha, \beta)=\limsup _{r \rightarrow \infty} \frac{\alpha(\log M(r, r, f))}{\beta(\log r)}, \\
& T(\alpha, \beta)=\limsup _{r \rightarrow \infty} \frac{\alpha(M(r, r, f))}{[\beta(r)]^{\rho(\alpha, \beta)}},
\end{aligned}
$$

where

$$
M(r, r, f)=\sup _{z, t \in \bar{D}_{R}}|f(t, z)|, \quad r<R
$$

In view of the concept introduced by MacLane [18] to the measures of order and type for an analytic function on a disk $|z|<R$, we normalize above definitions relative to the boundary under the transformation $r \rightarrow R /(R-r)$. Thus an analytic function $f(t, z) \in A\left(D_{R} \times D_{R}\right)$ with radial limits is said to be of generalized regular growth $\left(\rho_{o}(\alpha, \beta), T_{o}(\alpha, \beta)\right)$ if it satisfies

$$
\begin{aligned}
& \rho_{o}(\alpha, \beta)=\underset{r \rightarrow R}{\limsup } \frac{\alpha(\log M(r, r, f))}{\beta(R /(R-r))}, \\
& T_{o}(\alpha, \beta)=\limsup _{r \rightarrow R} \frac{\alpha(M(r, r, f))}{[\beta(R /(R-r))]^{\rho_{o}(\alpha, \beta)}}, \quad 1<r<R,
\end{aligned}
$$

where $\rho_{o}(\alpha, \beta)$ is referred to as the $(\alpha, \beta)$-order of $f(t, z)$ provided that $0<\rho_{o}(\alpha, \beta)<\infty$ and $T_{o}(\alpha, \beta)$ is referred to as the $(\alpha, \beta)$-type.

Example A. $f(t, z)=\exp \{(1 /(1-t))(1 /(1-z))\} \in A\left(D_{1} \times\right.$ $\left.D_{1}\right)$ has $(\alpha, \beta)$-order 2 and $(\alpha, \beta)$-type 1 for $\alpha(x)=\log x$ and $\beta(x)=x$.
In a neighborhood of origin, the function $f(t, z)$ has the local expansion

$$
f(t, z)=\sum_{n=0}^{\infty} \frac{w_{n}(t)}{n !} z^{n}
$$

where $w_{n}(t)=\left(\partial^{n} f / \partial z^{n}\right)(t, 0)$ is an entire solution of the ordinary differential equation

$$
t \frac{d^{2} w}{d t^{2}}+(\delta+1-t) \frac{d w}{d t}+n w=0
$$

where $w_{n}(t) z^{n}=n ! a_{n} L_{n}(\delta, t) z^{n}$. Following the method of Frobenius [19], a second independent solution of (9) can be obtained as

$$
X_{n}(\delta, t)=q L_{n}(\delta, t) \log t+\sum_{i=0}^{\infty} p_{i} t^{i}
$$

where $q \neq 0$ and $p_{i}$ are constants. So there exist $a_{n}$ and $b_{n}$ satisfying

$$
w_{n}(t)=n ! a_{n} L_{n}(\delta, t)+b_{n} X_{n}(\delta, t) .
$$

Because of the singularity of $X_{n}(\delta, t)$ at $t=0$, it leads to $b_{n}=0$. Thus

$$
f(t, z)=\sum_{n=0}^{\infty} a_{n} L_{n}(\delta, t) z^{n}
$$

Since $f(0, z)=\sum_{n=0}^{\infty} a_{n} L_{n}(\delta, 0) z^{n}$, we have the estimate $\left|w_{n}(0) z^{n}\right|=\left|n ! a_{n} L_{n}(\delta, 0) z^{n}\right| \sim n !\left|a_{n}\right|\left(n^{\delta} / \Gamma(\delta+1)\right) r^{n}$ for large $n$. Set

$$
\limsup _{n \rightarrow \infty} \frac{\alpha(n)}{\beta\left[n / \log \left(\left(n^{\delta} / \Gamma(\delta+1)\right)\left|a_{n}\right| R^{n}\right)\right]}=\mu(\alpha, \beta) .
$$

\section{Auxiliary and Main Results}

First we prove the following lemma.

Lemma 1. Let $f(t, z)=\sum_{n=0}^{\infty} a_{n} L_{n}(\delta, t) z^{n}$ and $f \in A\left(D_{R} \times\right.$ $\left.D_{R}\right)$. For every $1<r<R$, we set

$$
\begin{aligned}
\bar{M}(r, r, f) & =\sup _{n \in \mathbb{N}}\left\{\left\|a_{n} L_{n}(\delta, t)\right\| \cdot r^{n}, r>0\right\}, \\
\bar{\rho}_{o}(\alpha, \beta) & =\limsup _{r \rightarrow R} \frac{\alpha(\log \bar{M}(r, r, f))}{\beta(R /(R-r))} ;
\end{aligned}
$$

then

$$
\begin{aligned}
& \bar{\rho}_{o}(\alpha, \beta) \leq \mu(\alpha, \beta), \\
& \rho_{o}(\alpha, \beta) \leq \bar{\rho}_{o}(\alpha, \beta) .
\end{aligned}
$$

Proof. From (13), for $r$ sufficiently close to $R$, we have

$$
\log \left(\frac{n^{\delta}}{\Gamma(\delta+1)}\left|a_{n}\right| R^{n}\right) \leq \frac{n}{\beta^{-1}(\alpha(n) / \bar{\mu})}, \quad \bar{\mu}=\mu+\varepsilon
$$


or

$$
\begin{aligned}
& \log \left(\frac{n^{\delta}}{\Gamma(\delta+1)}\left|a_{n}\right| R^{n}\right) \\
& \quad \leq n \log \left(\frac{r}{R}\right)+\frac{n}{\beta^{-1}(\alpha(n) / \bar{\mu})} .
\end{aligned}
$$

Using the result

$$
\begin{array}{r}
\lim _{x \rightarrow \infty}\left|\frac{d\left(\beta^{-1}(c \alpha(x))\right)}{d(\log x)}\right| \leq b \log (1+\xi)=(1+o(\xi)), \\
\xi \longrightarrow 0,
\end{array}
$$

with the method of Calculus that, for every $r>1$ and $\mu>0$, the maximum of the function

$$
x \log \left(\frac{r}{R}\right)+\frac{x}{\beta^{-1}(\alpha(x) / \bar{\mu})}
$$

is reached at

$x$

$$
=\alpha^{-1}\left\{\bar{\mu} \beta\left[\frac{1-d \log \left(\beta^{-1}(\alpha(x) / \mu)\right) / d(\log x)}{\log (R / r)}\right]\right\},
$$

we get

$$
x=(1+o(1)) \alpha^{-1}\left(\bar{\mu} \beta\left(\frac{R}{(R-r)}\right)\right), \quad r \longrightarrow \infty .
$$

Now we have

$$
\begin{aligned}
\log \left(\frac{n^{\delta}}{\Gamma(\delta+1)}\left|a_{n}\right| r^{n}\right) & \leq C_{o} \alpha^{-1}\left(\bar{\mu} \beta\left(\frac{R}{(R-r)}\right)\right), \\
\log M(r, r, f) & \leq C_{o} \alpha^{-1}\left(\bar{\mu} \beta\left(\frac{R}{(R-r)}\right)\right) .
\end{aligned}
$$

Using the property of $\alpha$, we get

$$
\frac{\alpha(\log \bar{M}(r, r, f))}{\beta(R /(R-r))} \leq \bar{\mu} .
$$

Applying the limit supremum as $r \rightarrow R$, we obtain

$$
\bar{\rho}_{o}(\alpha, \beta) \leq \mu(\alpha, \beta) .
$$

Now consider

$$
f(t, z)=\sum_{n=0}^{\infty} a_{n} L_{n}(\delta, 0) z^{n}
$$

putting $r=\sqrt{r \cdot R} \sqrt{r / R}$ in the above, we get

$$
\begin{aligned}
M(r, r, f) \leq \sum_{n=0}^{\infty}\left|a_{n}\right|\left|L_{n}(\delta, t)\right|(\sqrt{r \cdot R})^{n}\left(\sqrt{\frac{r}{R}}\right)^{n}, & \left(\frac{r}{R}\right)<1,
\end{aligned}
$$

or

$$
\begin{aligned}
& M(r, r, f) \\
& \quad \leq \sum_{n=0}^{\infty} \sup \left(\left|a_{n}\right|\left|L_{n}(\delta, t)\right|(\sqrt{r \cdot R})^{n}\left(\sqrt{\frac{r}{R}}\right)^{n}\right),
\end{aligned}
$$

or

$$
\begin{aligned}
M(r, r, f) & \leq \bar{M}\left(r^{\prime}, r^{\prime}, f\right) \sum_{n=0}^{\infty}\left(\sqrt{\frac{r}{R}}\right)^{n} \\
& \leq \bar{M}\left(r^{\prime}, r^{\prime}, f\right)\left(\frac{1}{1-\sqrt{r / R}}\right), \\
r^{\prime} & =\sqrt{r \cdot R},
\end{aligned}
$$

$\log M(r, r, f) \leq \log \bar{M}\left(r^{\prime}, r^{\prime}, f\right)$

$$
-\log \left(1-\sqrt{\left(\frac{r}{R}\right)}\right)
$$

or

$$
\begin{aligned}
& \frac{\alpha(\log M(r, r, f))}{\beta(R /(R-r))} \\
& \leq \frac{\alpha\left(\log \bar{M}\left(r^{\prime}, r^{\prime}, f\right)-\log (1-\sqrt{(r / R)})\right)}{\beta(R /(R-\sqrt{r \cdot R}))} \\
& \quad \frac{\beta(R /(R-\sqrt{r \cdot R}))}{\beta(R /(R-r))} .
\end{aligned}
$$

Proceeding to limits, we obtain

$$
\rho_{o}(\alpha, \beta) \leq \bar{\rho}_{o}(\alpha, \beta) .
$$

Combining (24) and (30), we get

$$
\rho_{o}(\alpha, \beta) \leq \mu(\alpha, \beta) \text {. }
$$

Theorem 2. Let $f(t, z)=\sum_{n=0}^{\infty} a_{n} L_{n}(\delta, t) z^{n}$ such that

$$
\begin{aligned}
& \limsup _{n \rightarrow \infty} \frac{\alpha(n)}{\beta\left[n / \log \left(\left(n^{\delta} / \Gamma(\delta+1)\right)\left|a_{n}\right| R^{n}\right)\right]}=\mu(\alpha, \beta) \\
& \quad<\infty
\end{aligned}
$$

then $f$ is the restriction of analytic function in $\left(D_{R} \times D_{R}\right)(R>$ 1) and its $(\alpha, \beta)$-order $\rho(\alpha, \beta)=\mu(\alpha, \beta)$.

Proof. Since $f(0, z)=\sum_{n=0}^{\infty} a_{n} L_{n}(\delta, 0) z^{n}$ is an analytic function in $D_{R}$, we have

$$
\limsup _{n \rightarrow \infty}\left(\left|a_{n}\right| L_{n}(\delta, 0)\right)^{1 / n}=\frac{1}{R}, \quad R>1 .
$$

Using $L_{n}(\delta, 0)=n^{\delta} / \Gamma(\delta+1)$, we get

$$
\limsup _{n \rightarrow \infty}\left(\left|a_{n}\right|\right)^{1 / n}=\frac{1}{R}
$$


which is necessary and sufficient condition for $f \in A\left(D_{R} \times\right.$ $\left.D_{R}\right)$. So, for every $1<r<R$, the series $\sum_{n=0}^{\infty} a_{n} L_{n}(\delta, t) z^{n}$ is convergent in $D_{R} \times D_{R}$ when $\sum_{n=0}^{\infty} a_{n} L_{n}(\delta, t) z^{n}$ is analytic in $D_{R} \times D_{R}$.

Now we have to prove that $\mu(\alpha, \beta)$ is the $(\alpha, \beta)$-order of $f(t, z)$.

In order to complete the proof by Lemma 1, it is only to show that $\rho(\alpha, \beta) \geq \mu(\alpha, \beta)$. In view of the definition of $\rho(\alpha, \beta)$, we have, for every $\delta>0$, that there exists $1<r_{\varepsilon}<R$ such that, for every $r_{\varepsilon}<r<R$,

$$
\log M(r, r, f) \leq \alpha^{-1}\left[(\rho(\alpha, \beta)+\varepsilon) \beta\left(\frac{R}{(R-r)}\right)\right] .
$$

Using Cauchy's estimate of analytic functions, we have

$$
\left|\frac{\partial^{n} f}{\partial z^{n}}(0,0)\right| \leq n r^{-n} M(r, r, f)
$$

with the coefficients formula of the Taylor expansion,

$$
\frac{\partial^{n} f}{\partial z^{n}}(0,0)=a_{n} L_{n}(\delta, 0) n ! ;
$$

we get $M(r, r, f) \geq\left|a_{n}\right|\left|L_{n}(\delta, 0)\right| r^{n}$. Since $\left|L_{n}(\delta, 0)\right| \geq 1$, it gives

$$
\left|a_{n}\right| r^{n} \leq M(r, r, f) .
$$

Now using (35) in (38), we get

$$
\begin{aligned}
\log \left(\left|a_{n}\right| \frac{n^{\delta}}{\Gamma(\delta+1)} R^{n}\right) \\
\leq-n \log \left(\frac{r}{R}\right)+\delta \log n-\log (\Gamma(\delta+1)) \\
\quad+\alpha^{-1}\left[(\rho(\alpha, \beta)+\varepsilon) \beta\left(\frac{R}{(R-r)}\right)\right] .
\end{aligned}
$$

The minimum value of right-hand side is estimated at

$$
\begin{array}{r}
r=R\{1 \\
\left.-\frac{1}{\beta^{-1}\left((1 /(\rho+\varepsilon)) \alpha\left(n / \beta^{-1}(\alpha(n) /(\rho+\varepsilon))\right)\right)}\right\}, \\
\rho=\rho(\alpha, \beta) .
\end{array}
$$

Using the properties of functions $\alpha$ and $\beta$,

$$
\alpha\left(\frac{x}{\beta^{-1}(c \alpha(x))}\right) \simeq(1+o(1)) \alpha(x) ;
$$

for $c>0, x \rightarrow \infty$, and the properties of logarithm, we get

$$
\begin{aligned}
& \log \left(\left|a_{n}\right| \frac{n^{\delta}}{\Gamma(\delta+1)} R^{n}\right) \\
& \quad \leq C_{1}\left(\frac{n}{\beta^{-1}(\alpha(n) /(\rho+\varepsilon))}\right),
\end{aligned}
$$

where $C_{1}$ is a constant. Hence,

$$
\beta\left(\frac{C_{1} n}{\log \left(\left|a_{n}\right|\left(n^{\delta} / \Gamma(\delta+1)\right) R^{n}\right)}\right) \geq \frac{\alpha(n)}{(\rho+\varepsilon)} .
$$

Proceeding to limit supremum as $n \rightarrow \infty$, we obtain

$$
\mu(\alpha, \beta) \leq \rho(\alpha, \beta) .
$$

Hence the proof is completed.

Example B. $f(t, z)=\sum_{m+n=0}^{\infty}(1+m)^{l}(1+n)^{l} \exp \left\{m^{\mu} n^{\mu}\right\} t^{m} z^{n} \in$ $A\left(D_{1} \times D_{1}\right)$ has $(\alpha, \beta)$-order $(\mu /(1-\mu))$ for $\alpha(x)=\log x$ and $\beta(x)=x$, where $l$ is the positive integer.

Let $f(t, z)=\sum_{n=0}^{\infty} a_{n} L_{n}(\delta, t) z^{n}$ be analytic function of $(\alpha, \beta)$-order $\rho=\rho(\alpha, \beta)$ and write

$$
\begin{aligned}
T & (\alpha, \beta) \\
& =\limsup _{n \rightarrow \infty} \frac{\alpha(n)}{\left\{\beta\left[n / \log \left(\left(n^{\delta} / \Gamma(\delta+1)\right)\left|a_{n}\right| R^{n}\right)\right]\right\}^{\rho(\alpha, \beta)}} .
\end{aligned}
$$

Lemma 3. Let $f(t, z)=\sum_{n=0}^{\infty} a_{n} L_{n}(\delta, t) z^{n}$. For every $1<r<$ $R$,

$$
\bar{\sigma}_{1}(\alpha, \beta)=\limsup _{r \rightarrow R} \frac{\alpha(\log \bar{M}(r, r, f))}{[\beta(R /(R-r))]^{\rho(\alpha, \beta)}}
$$

then

$$
\sigma(\alpha, \beta) \leq \bar{\sigma}_{1}(\alpha, \beta) .
$$

Proof. Following the same reasoning as in the proof of Lemma 1, we obtain

$$
\begin{gathered}
\frac{\alpha(\log M(r, r, f))}{[\beta(R /(R-r))]^{\rho(\alpha, \beta)}} \\
\leq \frac{\alpha\left(\log \bar{M}\left(r^{\prime}, r^{\prime}, f\right)-\log (1-\sqrt{(r / R)})\right)}{[\beta(R /(R-\sqrt{r \cdot R}))]^{\rho(\alpha, \beta)}} \\
\quad \frac{[\beta(R /(R-\sqrt{r \cdot R}))]^{\rho(\alpha, \beta)}}{[\beta(R /(R-r))]^{\rho(\alpha, \beta)}} .
\end{gathered}
$$

Applying the limit supremum as $r \rightarrow R$, we get

$$
\sigma(\alpha, \beta) \leq \bar{\sigma}_{1}(\alpha, \beta) \text {. }
$$

Theorem 4. Let $f(t, z)=\sum_{n=0}^{\infty} a_{n} L_{n}(\delta, t) z^{n}$ be of finite generalized $(\alpha, \beta)$-order $\rho(\alpha, \beta)$ and

$$
\begin{aligned}
& T(\alpha, \beta) \\
& \quad=\limsup _{n \rightarrow \infty} \frac{\alpha(n)}{\left\{\beta\left[n / \log \left(\left(n^{\delta} / \Gamma(\delta+1)\right)\left|a_{n}\right| R^{n}\right)\right]\right\}^{\rho(\alpha, \beta)}} \\
& \quad<\infty .
\end{aligned}
$$

Then $f$ is the restriction of an analytic function in $\left(D_{R} \times\right.$ $\left.D_{R}\right)(R>1)$ and its $(\alpha, \beta)$-type $\sigma(\alpha, \beta)=T(\alpha, \beta)$. 
Proof. We have proven in Theorem 2 that $f$ is the restriction of an analytic function in $\left(D_{R} \times D_{R}\right)$. Now, in order to complete the proof, first we shall prove that $\sigma(\alpha, \beta) \leq T(\alpha, \beta)$. In view of the definition of $T$, for every $\varepsilon>0$, there exists $n \geq n_{\varepsilon}$ :

$\alpha(n)$

$$
\begin{array}{r}
\leq(T+\varepsilon)\left\{\beta\left[\frac{n}{\log \left(\left(n^{\delta} / \Gamma(\delta+1)\right)\left|a_{n}\right| R^{n}\right)}\right]\right\}^{\rho(\alpha, \beta)}, \\
T \equiv T(\alpha, \beta)<\infty, \\
\log \left(\left|a_{n}\right| \frac{n^{\delta}}{\Gamma(\delta+1)} R^{n}\right) \leq\left(\frac{n}{\beta^{-1}[(\alpha(n) /(\bar{T}))]^{1 / \rho}}\right), \\
\bar{T}=T+\varepsilon,
\end{array}
$$

since

$$
\begin{aligned}
& \log \left(\left|a_{n}\right| \frac{n^{\delta}}{\Gamma(\delta+1)} r^{n}\right) \\
& \quad \leq n \log \left(\frac{r}{R}\right)+\log \left(\left|a_{n}\right| \frac{n^{\delta}}{\Gamma(\delta+1)} R^{n}\right) .
\end{aligned}
$$

From inequality (51), we get

$$
\begin{aligned}
& \log \left(\left|a_{n}\right| \frac{n^{\delta}}{\Gamma(\delta+1)} r^{n}\right) \\
& \quad \leq n \log \left(\frac{r}{R}\right)+\left(\frac{n}{\beta^{-1}[(\alpha(n) /(\bar{T}))]^{1 / \rho}}\right) .
\end{aligned}
$$

For every $1<r<R$ and $r$ sufficiently close to $R$, we put

$$
\varphi(x, r)=x \log \left(\frac{r}{R}\right)+\left(\frac{x}{\beta^{-1}[(\alpha(x) /(\bar{T}))]^{1 / \rho}}\right) .
$$

The maximum value of $\varphi(x, r)$ is reached at

$$
\begin{aligned}
& x=x_{r} \\
& =\alpha^{-1}\left\{\left[\bar{T} \beta\left[\frac{1-d \log \left(\beta^{-1}(\alpha(x) / \bar{T})^{1 / \rho}\right) / d(\log x)}{\log (R / r)}\right]\right]^{\rho}\right\} .
\end{aligned}
$$

Using the relation

$$
\log \left(\frac{r}{R}\right)=\log \left(\frac{r-R}{R}+1\right) \sim \frac{r-R}{R}
$$

$($ as $(r-R) / R \rightarrow 0)$ and

$$
\left|\frac{d\left(\log \left(\beta^{-1}(\alpha(x) / \bar{T})^{1 / \rho}\right)\right)}{d(\log x)}\right| \leq k_{o},
$$

where $k_{o}$ is a positive constant, it gives

$$
x_{r}=(1+o(1)) \alpha^{-1}\left(\bar{T}\left(\beta\left(\frac{R}{R-r}\right)\right)^{\rho}\right) .
$$

Now, from relation (53), we have

$$
\log \left(\left|a_{n}\right| \frac{n^{\delta}}{\Gamma(\delta+1)} r^{n}\right) \leq \sup \varphi(x, r)
$$

or

$$
\begin{gathered}
\log \left(\left|a_{n}\right| \frac{n^{\delta}}{\Gamma(\delta+1)} r^{n}\right) \\
\leq \frac{(1+o(1)) \alpha^{-1}\left(\bar{T}(\beta(R /(R-r)))^{\rho}\right)}{R /(R-r)} .
\end{gathered}
$$

Since $R /(R-r)>1$, it gives

$$
\log \left(\left|a_{n}\right| \frac{n^{\delta}}{\Gamma(\delta+1)} r^{n}\right) \leq k_{1} \alpha^{-1}\left(\bar{T}\left(\beta\left(\frac{R}{R-r}\right)\right)^{\rho}\right) .
$$

Then

$$
\log M(r, r, f) \leq k_{1} \alpha^{-1}\left(\bar{T}\left(\beta\left(\frac{R}{R-r}\right)\right)^{\rho}\right)
$$

or

$$
\frac{\alpha(\log M(r, r, f))}{(\beta(R /(R-r)))^{\rho}} \leq \bar{T},
$$

or

$$
\sigma(\alpha, \beta) \leq T
$$

The inequality obviously holds for $T=\infty$. Now we shall prove that $\sigma(\alpha, \beta) \geq T(\alpha, \beta)$. Let $\sigma(\alpha, \beta)<\infty$. In view of definition of $\sigma(\alpha, \beta)$, we have, for every $\varepsilon>0$, that there exist $1<r_{\varepsilon}<R$, such that, for every $r>r_{\varepsilon}\left(R>r>r_{\varepsilon}>1\right)$,

$$
\log M(r, r, f) \leq \alpha^{-1}\left(\bar{\sigma}\left(\beta\left(\frac{R}{R-r}\right)\right)^{\rho}\right),
$$

$$
\bar{\sigma}=\sigma+\varepsilon .
$$

Now, using (51), we get

$$
\begin{aligned}
\log \left(\left|a_{n}\right| \frac{n^{\delta}}{\Gamma(\delta+1)} R^{n}\right) \\
\leq-n \log \left(\frac{r}{R}\right)+\delta \log n-\log (\Gamma(\delta+1)) \\
+\alpha^{-1}\left[\bar{\sigma}\left(\beta\left(\frac{R}{(R-r)}\right)\right)^{\rho}\right],
\end{aligned}
$$

or

$$
\begin{aligned}
& \frac{\log \left(\left|a_{n}\right|\left(n^{\delta} / \Gamma(\delta+1)\right) R^{n}\right)}{n} \leq-\log \left(\frac{r}{R}\right) \\
& +\frac{1}{n}[\delta \log n-\log (\Gamma(\delta+1)) \\
& \left.+\alpha^{-1}\left[\bar{\sigma}\left(\beta\left(\frac{R}{(R-r)}\right)\right)^{\rho}\right]\right],
\end{aligned}
$$


or

$$
\frac{1}{n} \log \left(\left|a_{n}\right| \frac{n^{\delta}}{\Gamma(\delta+1)} R^{n}\right) \leq(1+o(1)) \log \left(\frac{R}{r}\right),
$$

as $n \rightarrow \infty$. Now, using $\log (R / r) \sim(R-r) / r$ as $r \rightarrow R$, we have

$$
\begin{aligned}
& \frac{1}{n} \log \left(\left|a_{n}\right| \frac{n^{\delta}}{\Gamma(\delta+1)} R^{n}\right) \\
& \quad \leq(1+o(1)) \log \left(\left(\frac{R}{r}\right)-1\right) .
\end{aligned}
$$

Set

$$
\frac{r}{R}=\frac{\beta^{-1}[(\alpha(n) /(\bar{\sigma}))]^{1 / \rho}}{1+\beta^{-1}[(\alpha(n) /(\bar{\sigma}))]^{1 / \rho}}
$$

in above inequality, we get

$$
\frac{1}{n} \log \left(\left|a_{n}\right| \frac{n^{\delta}}{\Gamma(\delta+1)} R^{n}\right) \leq \frac{1}{\beta^{-1}[(\alpha(n) /(\bar{\sigma}))]^{1 / \rho}}
$$

or

$$
\frac{\alpha(n)}{\beta\left(n / \log \left(\left|a_{n}\right|\left(n^{\delta} / \Gamma(\delta+1)\right) R^{n}\right)\right)} \leq \bar{\sigma}=\sigma+\varepsilon .
$$

Applying the limit supremum as $n \rightarrow \infty$, we obtain

$$
\sigma(\alpha, \beta) \geq T(\alpha, \beta)
$$

The result is obviously true for $\sigma(\alpha, \beta)=\infty$. This completes the proof.

Example C. $f(t, z)=\sum_{m+n=0}^{\infty} \exp \left(\delta^{*}(m+n)^{\gamma^{*}}\right) t^{m} z^{n} \in A\left(D_{1} \times\right.$ $\left.D_{1}\right)$, where $0<\gamma^{*}<1$ and $0<\delta^{*}<\infty$, and has $(\alpha, \beta)$-order $\gamma^{*} /\left(1-\gamma^{*}\right)$ and $(\alpha, \beta)$-type $\left(1-\gamma^{*}\right)\left(\gamma^{*}\right)^{\gamma^{*} /\left(1-\gamma^{*}\right)}\left(\delta^{*}\right)^{1 /\left(1-\gamma^{*}\right)}$ for $\alpha(x)=\log x$ and $\beta(x)=x$.

\section{Conflicts of Interest}

The author declares that there are no conflicts of interest regarding the publication of this paper.

\section{References}

[1] P.-C. Hu and C.-C. Yang, "Global solutions of homogeneous linear partial differential equations of the second order," Michigan Mathematical Journal, vol. 58, no. 3, pp. 807-831, 2009.

[2] P.-C. Hu and C.-C. Yang, "A linear homogeneous partial differential equation with entire solutions represented by Bessel polynomials," Journal of Mathematical Analysis and Applications, vol. 368, no. 1, pp. 263-280, 2010.

[3] C. A. Berenstein and B. Q. Li, "On certain first-order partial differential equations in Cn," in Harmonic Analysis, Signal Processing and Complexity, vol. 238 of Progr. Math., pp. 29-36, Birkhauser, Boston, MA, USA, 2005.

[4] P. C. Hu and C.-C. Yang, "Malmquist type theorem and factorization of meromorphic solutions of partial differential equations," Complex Variables. Theory and Application. An International Journal, vol. 27, no. 3, pp. 269-285, 1995.
[5] P.-C. Hu and B. Q. Li, "On meromorphic solutions of nonlinear partial differential equations of first order," Journal of Mathematical Analysis and Applications, vol. 377, no. 2, pp. 881-888, 2011.

[6] B. Q. Li, "Entire solutions of certain partial differential equations and factorization of partial derivatives," Transactions of the American Mathematical Society, vol. 357, no. 8, pp. 3169-3177, 2005.

[7] B. Q. Li and E. G. Saleeby, "Entire solutions of first-order partial differential equations," Complex Variables. Theory and Application. An International Journal, vol. 48, no. 8, pp. 657-661, 2003.

[8] P. A. McCoy, "Approximation of pseudoanalytic functions on the unit disk," Complex Variables. Theory and Application. An International Journal, vol. 6, no. 2-4, pp. 123-133, 1986.

[9] D. Kumar, "Ultraspherical expansions of generalized biaxially symmetric potentials and pseudo analytic functions," Complex Variables and Elliptic Equations. An International Journal, vol. 53, no. 1, pp. 53-64, 2008.

[10] G. P. Kapoor and A. Nautiyal, "Growth and approximation of generalized bi-axially symmetric potentials," Indian Journal of Pure and Applied Mathematics, vol. 19, no. 5, pp. 464-476, 1988.

[11] D. Kumar and A. Basu, "Growth and L $\delta$-approximation of solutions of the Helmholtz equation in a finite disk," Journal of Applied Analysis, vol. 20, no. 2, pp. 119-128, 2014.

[12] X.-L. Wang, F.-L. Zhang, and P.-C. Hu, "A linear homogeneous partial differential equation with entire solutions represented by laguerre polynomials," Abstract and Applied Analysis, vol. 2012, Article ID 609862, 2012.

[13] M. N. Šeremeta, "On the connection between the growth of the maximum modulus of an entire function and the moduli of the coefficients of its power series expansion," in Twelve Papers on Real and Complex Function Theory, vol. 88 of American Mathematical Society Translations: Series 2, pp. 291301, American Mathematical Society, Providence, Rhode Island, 1970.

[14] S. M. Shah, "Polynomial approximation of an entire function and generalized orders," Journal of Approximation Theory, vol. 19, no. 4, pp. 315-324, 1977.

[15] G. S. Srivastava and S. Kumar, "On approximation and generalized type of entire functions of several complex variables," European Journal of Pure and Applied Mathematics, vol. 2, no. 4, pp. 520-531, 2009.

[16] S. B. Vakarchuk and S. I. Zhir, "On the best polynomial approximation of entire transcendental functions of generalized order," Ukrainian Mathematical Journal, vol. 60, no. 8, pp. 1183-1199, 2008.

[17] S. B. Vakarchuk and S. I. Zhir, "On the best polynomial approximations of entire transcendental functions of many complex variables in some Banach spaces," Ukrainian Mathematical Journal, vol. 66, no. 12, pp. 1793-1811, 2015.

[18] G. R. MacLane, "Asymptotic values of holomorphic functions," Rice University Studies, vol. 49, no. 1, 83 pages, 1963.

[19] Z. X. Wang and D. R. Guo, Special Functions, World Scientific, River Edge, NJ, USA, 1989. 


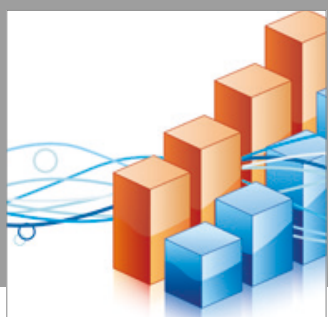

Advances in

Operations Research

vatersals

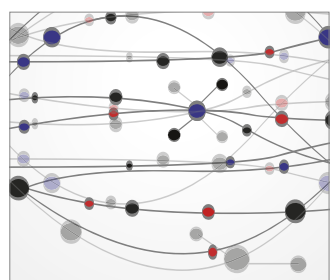

\section{The Scientific} World Journal
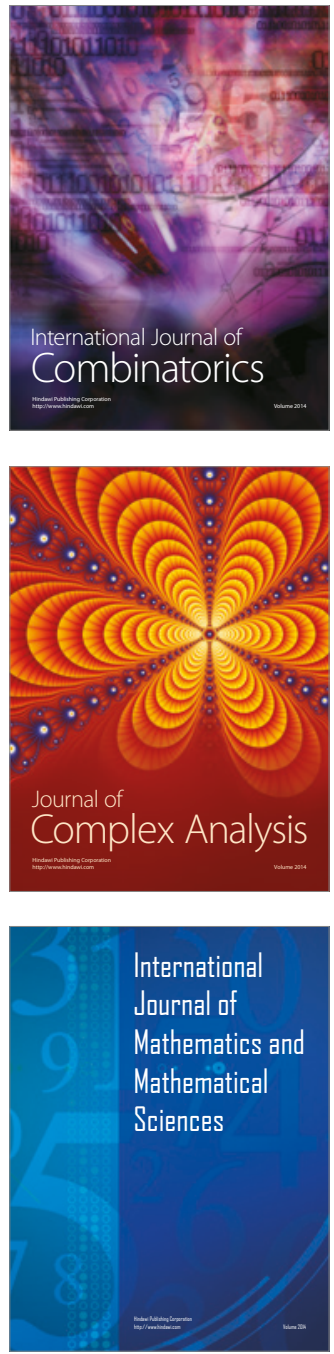
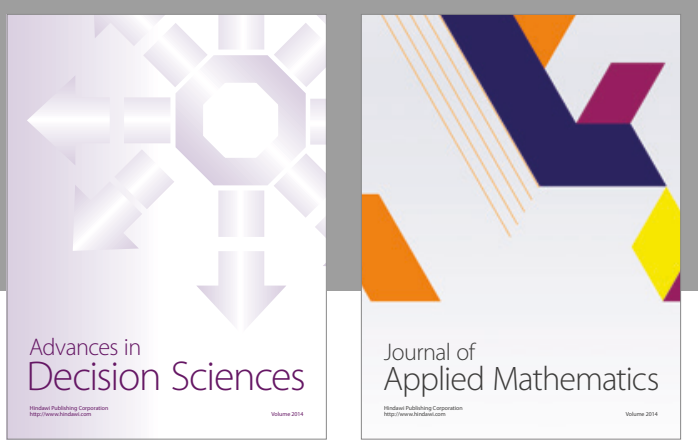

Algebra

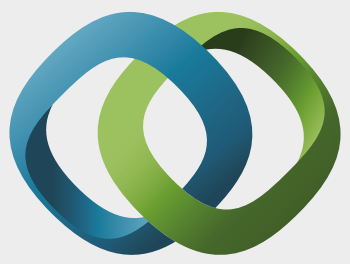

\section{Hindawi}

Submit your manuscripts at

https://www.hindawi.com
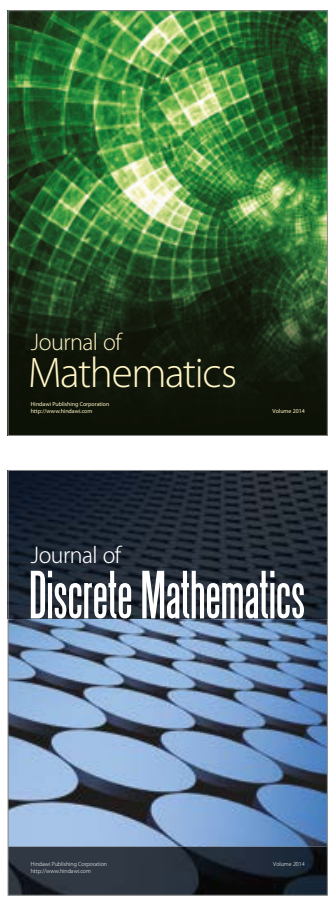



Mathematical Problems in Engineering


Journal of

Function Spaces

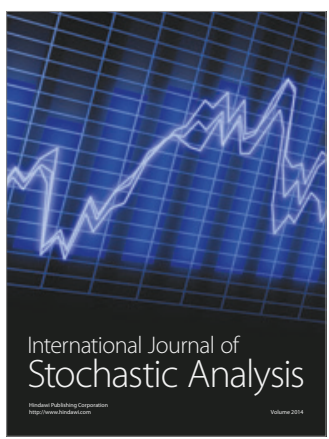

Probability and Statistics
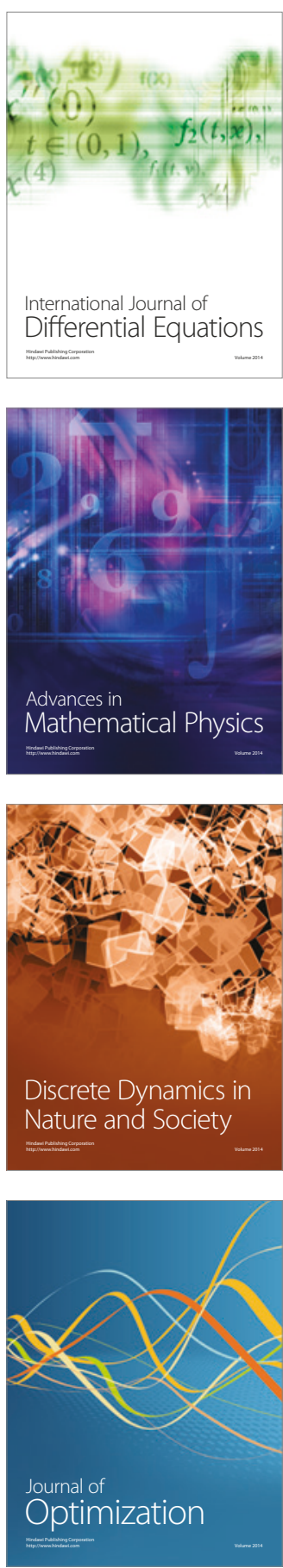